\title{
Facile synthesis of graphene via direct water-sodium dodecylbenzenesulfonate exfoliation
}

\begin{abstract}
In this study, a mild, one-step electrochemical exfoliation was demonstrated for the synthesis of graphene with the assistance of water and a surfactant, particularly sodium dodecylbenzenesulfonate and sodium dodecyl sulphate. Different types of water-surfactant solutions in different concentrations could influence the exfoliation of graphite rods. From one to several layers of graphene flakes (with a thickness of approximately $1 \mathrm{~nm}$ ) could be produced directly after sonication. AFM images showed that the flake diameters from this source were typically small. Raman and IR spectroscopic analyses of the dispersed phase suggested that the exfoliation of graphene sheets was accomplished. The flakes were also characterized using field emission scanning electron microscopy, X-ray diffraction, and cyclic voltammetry techniques. Further improvements in this methodology may pave the way to develop green, cost-effective, and large-scale production methods for graphene sheets.
\end{abstract}

Keyword: Graphene; Carbonaceous material; Surfactant; Electrochemical exfoliation 